九州大学学術情報リポジトリ

Kyushu University Institutional Repository

Simultaneous measurement method of pressure and temperature using dual layer PSP/TSP with lifetime based method

文，吉周

九州大学大学院工学府機械工学専攻

森，英男

九州大学大学院工学研究院機械工学部門

古川，雅人

九州大学大学院工学研究院機械工学部門

ht tp://hdl. handle. net/2324/4110740

出版情報：Measurement science \& technology. 29 (125301), pp.1-13，2018-10-30. IOP Publishing バージョン：

権利関係 : 


\section{Simultaneous measurement method of pressure and temperature using dual-layer PSP/TSP with lifetime-based method}

Kil-Ju Moon(1), Hideo Mori( ${ }^{(1)}$ and Masato Furukawa(1)

(1) Department of Mechanical Engineering, Kyushu University, 744 Motooka, Nishi-ku, Fukuoka, 819-0395, Japan

Keywords : Pressure sensitive paint, Temperature sensitive paint, dual-layer PSP/TSP, lifetime-based method 


\begin{abstract}
Recently, the application field of pressure measurement technique using pressure sensitive paint (PSP) has been expanding. However, temperature dependence of PSP is one of a serious obstacle to accurate pressure measurement in low gauge pressure condition because PSP is absolute pressure sensor. Therefore, temperature information of PSP is indispensably required for accurate pressure measurement, especially in low gauge pressure condition. As a result, both temperature information and pressure information with a function of temperature are required simultaneously to use the PSP in low gauge pressure condition. Dual-layer PSP/TSP (DL-PTSP) is one solution, but simultaneous measurement of pressure and temperature requires two optical filters to separate luminescence of PSP and TSP, resulting in complex equipment and additional error caused by parallax. To solve the problem, we introduce lifetime-based method for DL-PTSP that allows simultaneous measurement of pressure and temperature. It is a method to separate each component of luminescence with different lifetimes. At first, The DL-PTSP developed in this study was optimized by reducing the luminescence intensity of the PSP with longer lifetime while maintaining the luminescence intensity of the TSP with shorter lifetime, considering the lifetime measurement method. Secondly, we have clarified the optimum time conditions the monolayer TSP and the monolayer PSP by the analysis of their luminescence decay process, by controlling the irradiation delay of light source relative to camera shutter. Thirdly, sensitivity of the DL-PTSP using the conditions optimized for mono-layer TSP and PSP was examined. The luminescence decay of the TSP component shows sufficient temperature dependence, while pressure dependence is very small. On the other hand, the dependence of the luminescence decay of the PSP component on temperature and pressure is almost equal to that of the mono-layer PSP. The results confirm the feasibility of simultaneous measurement of pressure and temperature using DLPTSP.
\end{abstract}




\section{Introduction}

Pressure sensitive paint (PSP) and temperature sensitive paint (TSP) are useful measurement method for measuring pressure and temperature of solid surfaces [1] [2]. Bell et al.[3] have reported on the different measurement systems and procedures using PSP. Recently, the application fields of PSP and TSP have been extended to low gauge pressure distribution measurement field by development of camera and measurement technology[4]. Ibrahim et al. [5] have reported on visualization of the subsonic jet-plate impingement and surface pressure of rotating blades using the PSP. Peng et al. [6] have reported the study on wall pressure fluctuation in low speed flows using fast PSP. Bell [7] and Jeong et al.[8] have visualized the pressure distribution on the Lockman wing surface and delta wing respectively in low speed flow field. However, temperature dependence, which is one of the most serious disadvantage of PSP, causes relatively larger errors at low gauge pressures condition because PSP is absolute pressure sensor[9].

To compensate for the temperature dependence of PSP, there have been many reports of temperature correction methods of PSP. Mitsuo et al.[10] have reported the temperature correction of PSP using temperature measurement by IR camera. However, it is difficult to apply this method to internal flows, because most of optical glass materials for windows of a wind tunnel, which is transmissible for visible light and UV, does not transmit IR emission. Nakakita et al.[11] applied a method of painting PSP and TSP on each side of airplane models for temperature correction of the PSP. However, the method has restrictions that both the shape of the object and the flow field must be perfectly symmetric. Nowadays, approaches to combine PSP and TSP have been studied[12]. One approach is to develop bi-luminophore paint combining pressure-sensitive luminophore with pressure-insensitive but temperature-sensitive luminophore. Goss et al. [13] reported the bi-luminophore paint technique with the separation of luminescence of two luminophores using the difference of lifetime. In this case, mixing pressure-sensitive luminophore and temperature-sensitive luminophore often causes interference between the two kinds of luminescent molecules, resulting in decrease of pressure sensitivity of PSP or luminescence intensity, and photostability of PSP and TSP. Therefore, the combination of luminescent molecules suitable for bi-luminophore paint is very limited. Mochizuki et al. [14] developed the method to introduce luminescent molecules into porous nanoparticles, in order to avoid the interaction. Egami et al. [15] and Peng et al. [16] reported the grid-pattern spray technique using inkjet technology for simultaneous measurement of pressure and temperature. In this case, there is a limitation that spraying equipment is expensive and can only be applied to flat surfaces. Hyakutake et al. [17] reported another simpler approach, stacking a PSP layer and a TSP layer. In this approach, pressure-sensitive luminescent molecules do not contact physically with temperature-sensitive luminescent molecules, and the effect of the interaction between the two luminophores are relatively small. Moon et al. [18] reported the effect of temperature compensation of PSP using this approach, 
for application in low-speed flows with very small gauge pressure below 1kPa. Mori et al. [19] applied the method to measurement of pressure distribution on a sidewall in a supersonic flow passing through a turbine cascade. In this paper, this method is called as dual-layer PSP/TSP (DL-PTSP). However, "simultaneous" measurement of pressure and temperature needs multiple cameras with different optical filter, or a stereo-viewer to divide pixels of a camera. The former method raises the initial cost to introduce multiple cameras, and the latter method decreases the spatial resolution of the images. In addition, the both methods are affected by parallax of the two sets of images.

Nowadays lifetime-based imaging measurement using PSP have been reported. Above-mentioned report by Goss et al. [13] applies the lifetime-based method to bi-luminophore paint, in order to decrease the temperature sensitivity in pressure measurement. In addition, Mitsuo et al. [20] reported temperature compensation effect of PSP using luminescence lifetime decay and multiple gated times of CCD camera. In this case, temperature compensation effect is reported only in the high speed flow (high gauge pressure) condition. Therefore, we propose to apply lifetime-based method to DL-PTSP, which has the high temperature compensation ability and high pressure sensitivity even in low gauge pressure condition. Because the lifetime-based method enables to separate the luminescence of PSP and that of TSP using the difference of their lifetimes, the method allow simultaneous measurement of pressure and temperature with a single camera, even in relatively low gauge pressure condition. In this study, DL-PTSP is composed of a porphyrin-based PSP with relatively long lifetime and a ruthenium-complex-based TSP with relatively short lifetime. We investigate the lifetime characteristics of the mono-layer TSP and PSP, both are the components of the DL-PTSP. The results are examined to propose the method to separate the luminescence of DL-PTSP. Finally, we examine the feasibility of simultaneous measurement using the DL-PTSP and the lifetime-based method. 


\section{Principle of lifetime-based method}

The lifetime-based method is one of the measurement method of pressure and temperature by PSP and TSP using the dependence of the luminescence lifetime on pressure and temperature. The greatest advantage of the lifetime-based method is that it is not affected by non-uniform irradiation of PSP or TSP, resulting in higher accuracy than the intensity-based method. The quantum yield $\Phi$ of luminescence of PSP or TSP is defined by Eq.(1)

$$
\Phi=\frac{\text { rate of liminescence }}{\text { rate of excitaion }}=\frac{\kappa_{r}}{\kappa_{r}+\kappa_{n r}+\kappa_{q}[Q]}=\frac{I}{I_{a}},
$$

Where, $\kappa_{r}$ is rate constant for the radiation process, $\kappa_{n r}$ is rate constant for non-radiative processes $\kappa_{q}$ is rate constant for the quenching process and [Q] is the population of the quencher. $I$ is the luminescence intensity and $I_{a}$ is the intensity of light absorption. Where, the sum of rate constants of the denominator of quantum yield correspond to the inverse of the lifetime of luminophore molecules.

$$
\tau=\frac{1}{\kappa_{r}+\kappa_{n r}+\kappa_{q}[Q]}
$$

The relationship between the luminescence intensity $(I)$ and time $(t)$ in a one-dimensional model can be expressed by the following Eq.(3),

$$
I(t)=I\left(t_{r e f}\right) \exp \left(-\frac{t-t_{r e f}}{\tau}\right)
$$

where $t_{r e f}$ is the "reference" time. As is expressed in Eq. (3), the luminescence decay profile $I(t) / I\left(t_{\text {ref }}\right)$ is directly related to the lifetime $\tau$, and therefore the dependence of $\tau$ on pressure and temperature causes the dependence of $I(t) / I\left(t_{r e f}\right)$ on pressure and temperature. In this study, we discuss the dependence of luminescence decay characteristics of the luminophore measured by a camera on pressure and temperature instead of directly discussing the dependence of the lifetime $\tau$ of the luminophore itself. The luminescence decay characteristics is evaluated by using a linear empirical formula relating $I(t) / I\left(t_{r e f}\right)$ and pressure or temperature, as shown in Eq. (4) and (5).

$$
\begin{gathered}
\frac{I(t)}{I\left(t_{\text {ref }}\right)}=A_{0}+A_{1} \frac{P}{P_{\text {ref }}}, \\
\frac{I(t)}{I\left(t_{\text {ref }}\right)}=B_{0}+B_{1} \frac{T}{T_{\text {ref }}},
\end{gathered}
$$

where $P_{\text {ref }}$ and $T_{\text {ref }}$ are known "reference" absolute pressure and absolute temperature, respectively. 


\section{Structure of DL-PTSP and optimization}

Fig.1 shown schematic diagram of dual-layer pressure and temperature sensitive paint (DL-PTSP). The main idea of the DL-PTSP structure is stacking up layers as can be seen in Fig.1. A TSP layer using binder without oxygen permeability is placed as the lower layer, and a PSP layer which need oxygen response is placed as the upper layer. An intermediate layer was placed to prevent mixing and interference of luminophore layers (TSP and PSP layer) by separating them each other. It also works to completely prevent oxygen permeating into the TSP layer. The DL-PTSP has two intermediate layers; the 1st intermediate layer is located between a white paint layer at the bottom acting as a screen and the TSP layer, and the 2nd intermediate layer is located between the TSP layer and the PSP layer as is mentioned previously. The 1 st intermediate layer is composed of polyurethane resin, which is same as the binder of the TSP layer, in order to prevent the mixing of the white paint and the TSP layer. The 2nd intermediate layer is composed of three sublayers, over-coating of TSP layer, oil based clear paint and base-coating of PSP layer. The over-coating of the TSP layer made of polyurethane, which is same as the TSP binder, and the base-coating of the PSP layer made of poly[1-(trimethylsilyl)-1propyne] (poly(TMSP)), which is the same as the PSP binder, play a role in preventing mixing of the polymers of the TSP or the PSP with the oil-based clear paint. The oil-based clear paint separates the two luminophore layers, and prevents permeation of oxygen molecules into the TSP layer. By placing the intermediate layers in the DL-PTSP, the PSP and TSP layers work properly without interfering each other. Because the two luminescent layers of DL-PTSP emit luminescence with the different lifetime, it can be separated by using the difference of the lifetime.

In this study, dichlorotris(1,10-phenanthroline)ruthenium(II) hydrate (Ru-phen), whose lifetime is of the order of $\mu \mathrm{s}$, is chosen as a luminophore of TSP, and Pt(II) meso-tetra(pentafluorophenyl) porphine (PtTFPP), whose lifetime is about one-order longer than that of Ru-phen. Luminophore and binder used in this study are set out in the Table.1 with the lifetime of each luminophore.

Fig.2 shows cross sectional view of DL-PTSP by scanning electron microscope (SHIMADZU, SS550). It was confirmed that the total thickness of the paint was about $100 \mu \mathrm{m}$. In detail, white paint layer was $20 \mu \mathrm{m}$, TSP layer including polyurethane base-coating and over-coating was $60 \mu \mathrm{m}$ and PSP layer including clear paint and poly(TMSP) layer for base-coating was $20 \mu \mathrm{m}$. However, the luminophore layer formed a very thin film with a few $\mu \mathrm{m}$.

First of all, it is necessary to optimize the ratio of the luminophore intensity of the PSP layer and the TSP layer of the DL-PTSP, because the imbalance between the luminescence intensity of the TSP layer and that of the PSP layer may cause the decrease in the sensitivity of the TSP. For example, if the luminescence intensity of the PSP layer is higher than that of the TSP layer at a few microseconds after the LED pulse is turned off, which is approximately equal to the lifetime of Ru-phen, it may decrease the temperature sensitivity of the TSP drastically. Therefore, to consider the application of 
DL-PTSP, it is necessary to adjust the intensity of the TSP layer and the PSP layer to the optimum level. It is advantageous if the luminescence intensity of the TSP layer with relatively short lifetime is higher than that of the PSP layer with long lifetime for the application of DL-PTSP using lifetimebased method. Therefore, we employ a method to reduce the intensity of the PSP layer with keeping the intensity of the TSP. However, over-reduction of the luminescence intensity of the PSP layer results in low SN ratio, therefore luminescence intensity of the PSP layer must be optimized. The PSP used in this study was optimized by controlling the luminophore concentration in the binder, spread volume and light source irradiation time in the PSP used in the previous study [18]. Table.2 shows the amount of luminophore and binder for DL-PTSP. The amounts shown in Table. 2 are based on the sample size $(4 \mathrm{~cm} \times 4 \mathrm{~cm})$ commonly used in this study. The spray amount was reduced to half and the binder concentration of the PSP was 5 times higher than that of PSP used in the previous study [18]. The intensity of PSP is determined as the irradiation time of the excitation light (LED). In this study, irradiation time was determined to be $15 \mu$ s, considering the minimum time for complete excitation of TSP. In this way, the intensity of the PSP was reduced to about $10 \%$ of that of the conventional monolayer PSP. The intensity difference between TSP and PSP was set to be 3:1 at the temperature measurement time $(t=5 \mu \mathrm{s})$. It is important to find an appropriate intensity ratio between TSP and PSP because that it is trade-off relationship between temperature sensitivity of TSP and SN ratio of PSP in DL-PTSP.

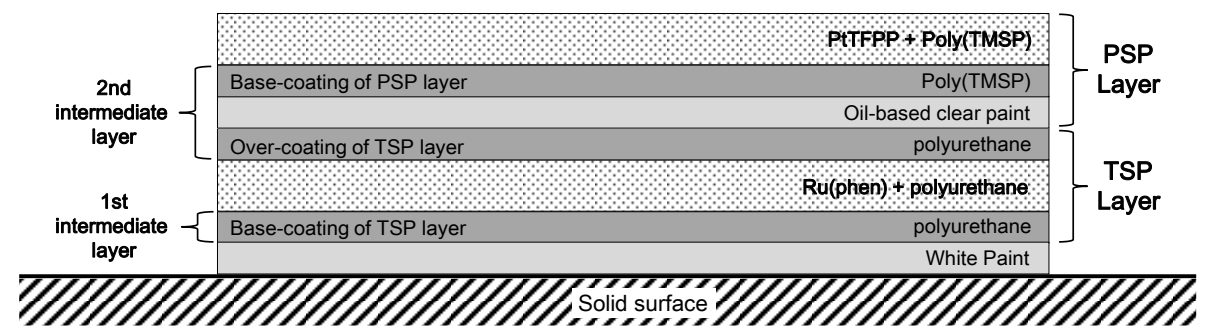

Fig.1 Schematic diagram of DL-PTSP

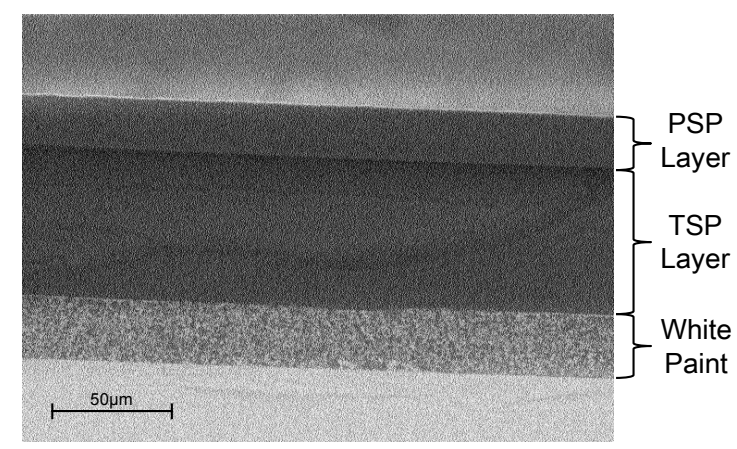

Fig.2 Cross sectional view of DL-PTSP 
Table.1 formulations and lifetime of PSP and TSP

\begin{tabular}{|c|c|c|c|}
\hline & luminophore & binder & lifetime $^{[2]}$ \\
\hline PSP & PtTFPP & Poly(TMSP) & $50 \mu \mathrm{s}$ \\
\hline TSP & Ru(phen) & polyurethane & $4.7 \mu \mathrm{s}$ \\
\hline
\end{tabular}

Table.2 Amount of luminophore and binder for DL-PTSP

\begin{tabular}{|c|c|c|c|}
\hline & luminophore & binder & Solvent \\
\hline PSP & $0.4 \mathrm{mg}$ & $8.0 \mathrm{mg}$ & Toluene $1.0 \mathrm{ml}$ \\
\hline TSP & $2.0 \mathrm{mg}$ & $1.6 \mathrm{ml}$ & Ethanol $0.2 \mathrm{ml}$ \\
\hline
\end{tabular}




\section{Experimental apparatus and sample}

A schematic diagram of the experimental apparatus is shown in Fig.3. The calibration chamber was made of acrylic resin which can transmit both excitation light and emission light. The pressure in the calibration chamber was monitored by an electric pressure gauge (Keyence, AP-C35) and controlled by syringes. The error is within $\pm 0.1 \mathrm{kPa}$ of the set pressure value. The temperature of a sample installed in the calibration chamber was controlled by a peltier PID controller (CELL System, TDC1020) with a platinum resistance thermometer sensor (JIS Pt100 Class A, compatible with IEC 60751) connected to the controller. The error is within $\pm 0.1 \mathrm{~K}$ of the set temperature value. A monochrome high speed camera with 12bit gradation (Photron, FASTCAM SA 5) was used for taking sample images. The frame rate of the camera was set to $100 \mathrm{kfps}(10 \mu \mathrm{s})$ and the exposure time of each image was 1 /frame. (In the case of FASTCAM SA5 exposure time set to 1 /frame, death time is $0.66 \mu \mathrm{s}$ ) The resolution was set to $64 \times 64$ pixels, all of which were located on the luminescent region of the sample, and the luminescence intensity was averaged over the all pixels. A 540nm long-pass filter was attached in front of the camera lens to block stray light from the light source. And, high-power LED (Luminus, CBT-120-UV-C31-P382-22) with a peak wavelength of $385 \mathrm{~nm}$ was used in order to excite the two type of luminophore used in this study. The reason for choosing the wavelength is that the absorption band of the PSP is narrow while the absorption band of the TSP is relatively wide and the LED covers the both absorption bands. The LED is operated in pulse irradiation mode by using external signals, and the fall time was set at $2 \mu \mathrm{s}$. A function generator (LeCroy, wavestation2012) was used as a source of the pulse signal for the LED. The function generator generates rectangular waves with a period of $100.2 \mu \mathrm{s}$ and the duty ratio of $15 \%$, that is, irradiation time per period is $15.0 \mu \mathrm{s}$. The function generator was synchronized with the camera by "measurement start" trigger of the camera in order to minimize temperature change from LED irradiation. The operation signal of camera and function generator were recorded in DAQ (CONTEC, AI-1204Z-PCI) for post processing (image rearrangement). The sampling rate of DAQ was $5 \mathrm{MHz}$. Post processing was done using MATLAB.

In this study, three types of samples were tested; mono-layer TSP, mono-layer PSP, and DL-PTSP. As is mentioned in the previous section, the imbalance in the luminescence intensity of the TSP layer and the PSP layer may cause the decrease in the sensitivity of the TSP component or the PSP component of the DL-PTSP. Therefore, it is important to clarify the characteristics of the TSP and the PSP, especially the dominant parameter to control the luminescence intensity, as well as the pressure sensitivity and the temperature sensitivity, by analysis of the mono-layer TSP and the monolayer PSP. For analysis of the mono-layer samples, temperature sensitivity is tested for the temperature range from $18.0^{\circ} \mathrm{C}$ to $22.0^{\circ} \mathrm{C}(291.2 \mathrm{~K}$ to $295.2 \mathrm{~K})$ in increments of $1.0 \mathrm{~K}$ at atmospheric pressure condition, which was $102.0 \mathrm{kPa}$, while pressure sensitivity is tested for the pressure range from $-8.0 \mathrm{kPa}$ to $8.0 \mathrm{kPa}$ gauge in increments of $4.0 \mathrm{kPa}$ at $20.0^{\circ} \mathrm{C}(293.2 \mathrm{~K})$. On the other hand, for analysis of the 
DL-PTSP, both the temperature and pressure are variable parameters for the total 25 conditions.

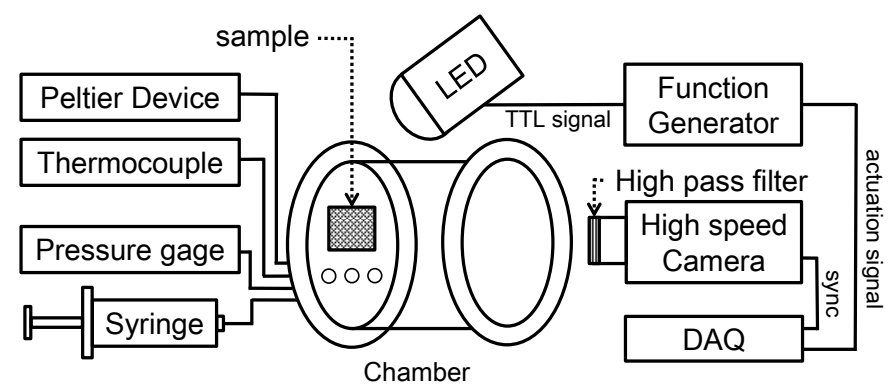

Fig.3 Schematic diagram of the experimental apparatus 
5. Shift irradiation measurement method to measure the luminescence decay characteristics using high speed camera

Luminophores used in this study have lifetimes of the order of microseconds or tens of microseconds, as can be seen in Table.1. It is difficult to take sequential images of luminescence decay of Ru-phen, which is used as the luminophore of the TSP, using a camera, because taking images with high frame rate and exposure time shorter than the lifetime results in very low SN ratio. Generally, lifetime-based method using a high-speed camera is taking images of two or three time gates, each has width of the order of microseconds. In this case, the width and the start time of each gate must be determined prior to the application, and therefore the dependence of the lifetime on pressure or temperature must be known. In this study, we devised a new method to obtain decay curve of the luminescence of luminophores by shifting the delay time of camera gate relative to the irradiation timing of the light source. The method is called as 'shift irradiation measurement method', abbreviated as 'SIM method' in this paper. Fig. 4 shows the sequence of SIM method for the condition in this study. The repetition period of the light source was set at $100 \mu$ s, with the duty ratio of $15 \%$, plus the delay time for each period of $0.2 \mu \mathrm{s}$, totally $100.2 \mu \mathrm{s}$. The sampling time of the DAQ to record the operating signal of the camera and the light source was set at $0.2 \mu \mathrm{s}$, which is equal to the time shift for each irradiation period. As a result, the timing of the exposure gate of the camera relative to the irradiation pulse is shifted by $0.2 \mu$ s for each irradiation period, as shown in Fig.4, and the timing for the 51 st irradiation is equal to that for the 1st irradiation. By reordering the 500 images taken for 50 series of irradiations, images of luminescence decay with the time interval of $0.2 \mu$ s is obtained. In this study, the process mentioned above is repeated 32 times, and the 32 sets of the images are averaged to increase the $\mathrm{SN}$ ratio.

In this study, time $t=0 \mu \mathrm{s}$ is defined as the instant when the rectangular signal for the light source (LED) from the function generator falls. In addition, luminescence intensity was normalized by that at time $t_{r e f}=2 \mu \mathrm{s}$, which is equal to the fall time of the LED used as the light source. On the other hand, for the DL-PTSP, the two types of the normalization was performed, by using the intensity at $t_{r e f}=2 \mu \mathrm{s}$ and $t_{r e f}=26 \mu \mathrm{s}$. Because the time of $26 \mu \mathrm{s}$ is much larger than the lifetime of Ru-phen, the luminophore of the TSP and the contribution of the TSP layer to the luminescence intensity at $t_{r e f}=26 \mu \mathrm{s}$ is negligible, and therefore the normalization at $t_{r e f}=26 \mu$ s can eliminate the effect of TSP layer for pressure measurement using the PSP layer of DL-PTSP. By applying the SIM method to the lifetime based method, the systematic error can be reduced and the existing equipment can be utilized. 


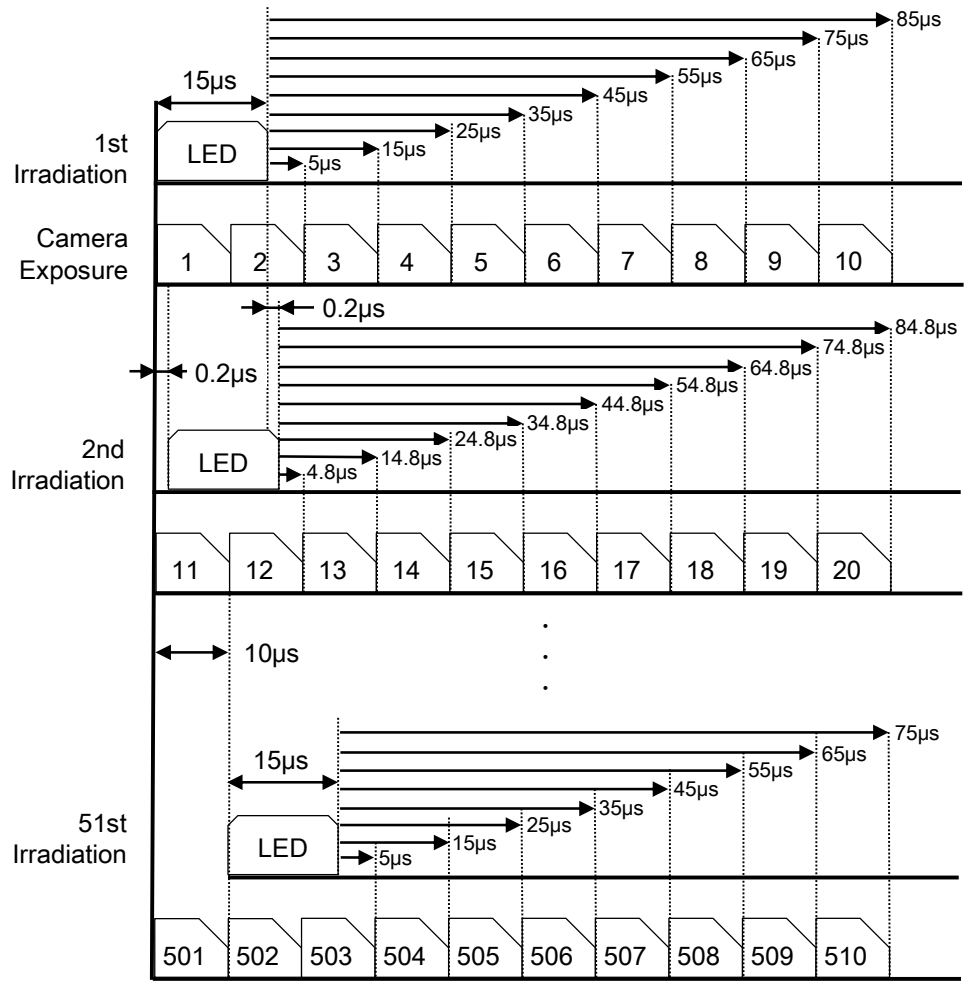

Fig.4 Sequence of shift irradiation measurement method 


\section{Characteristics of mono-layer TSP and mono-layer PSP}

\subsection{Mono-layer TSP}

Fig.5-1 and Fig.6-1 show the dependence of the luminescence intensity of the mono-layer TSP for a period of irradiation, including luminescence decay and excitation, on pressure and temperature, respectively, using SIM measurement method. The horizontal axes indicate the time $t$ when the gate of the camera exposure starts, and the vertical axes indicate the luminescence intensity averaged over $64 \times 64$ pixels. In the case of the mono-layer TSP, it can be confirmed that luminescence intensity falls close to zero at $t \sim 20 \mu \mathrm{s}$. Although the luminescence intensity when the excitation light is on is over the range (maximum value of the intensity is $2^{12}-1=4095$ ) of the camera, the intensity at $t=0$ is under the maximum. The saturation of the luminescence intensity during the excitation does not affect the reliability of the experimental results, because this study is focused on the luminescence decay characteristics, and it was confirmed from manufacturer that the saturation of the camera does not damage the camera. As previously stated, the higher intensity of TSP is advantageous for improving sensitivity and SN ratio of DL-PTSP. Fig.5-2 and Fig.6-2 also show the dependence of the luminescence decay characteristics on pressure and temperature, but the luminescence intensity shown in the vertical axes is normalized by that at the "reference" time $t_{r e f}=2 \mu \mathrm{s}$, and the range of the horizontal axes is set as $2 \mu \mathrm{s} \leq t \leq 20 \mu \mathrm{s}$, showing the decay process of the luminescence only. The results shown in Fig.5-2 and Fig.6-2 state that mono-layer TSP has temperature dependence, while it does not show pressure dependence. Fig.5-4 and Fig.6-4 show the relationships between normalized luminescence $I(t=5 \mu \mathrm{s}) / I\left(t_{r e f}\right)$ and normalized pressure or temperature, respectively, following to Eq. (4) and (5). The reference pressure and temperature used for the normalization is $102.0 \mathrm{kPa}$ and $293.15 \mathrm{~K}$, respectively and the error bars are indicated by standard deviation for each condition. The gradient $B_{1}$ of Eq. (5) shown in Fig. 6-4 is -2.157, which is high enough for temperature measurement, while the gradient $A_{1}$ of Eq. (4) corresponding to the pressure dependence shown in Fig.5-4 is -0.011, which is negligibly small. Fig.5-3 and Fig.6-3 show the time dependence of the gradient $A_{1}$ and $B_{1}$ of Eq. (4) and (5), respectively. As depicted in Fig.6-3, the magnitude of the gradient $\left|B_{1}\right|$ showing the temperature dependence is maximum at $t=5 \mu \mathrm{s}$, that is, $t-t_{r e f}=3 \mu \mathrm{s}$. On the other hand, Fig. 5-3 indicates that the gradient $\left|A_{1}\right|$ corresponding to the pressure dependence is very small for $2 \mu \mathrm{s} \leq$ $t \leq 20 \mu \mathrm{s}$. As a result, mono-layer TSP has high temperature sensitivity by using Eq. (5) for $t-$ $t_{r e f}=3 \mu \mathrm{s}$, and negligibly small pressure sensitivity. 


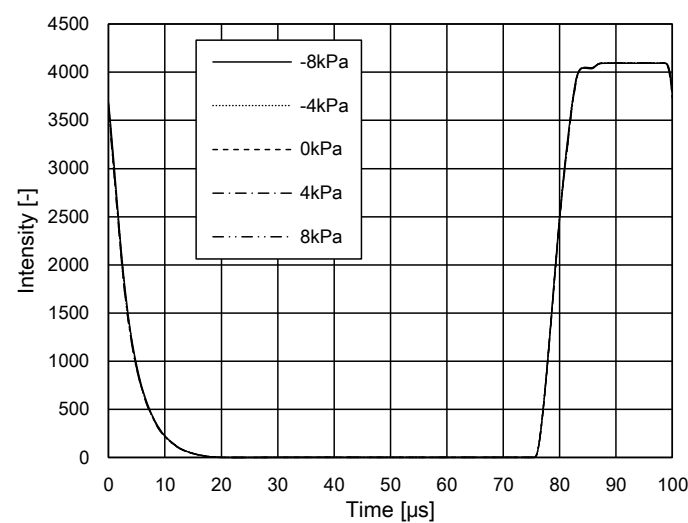

Fig.5-1 Luminescence intensity for a period of irradiation

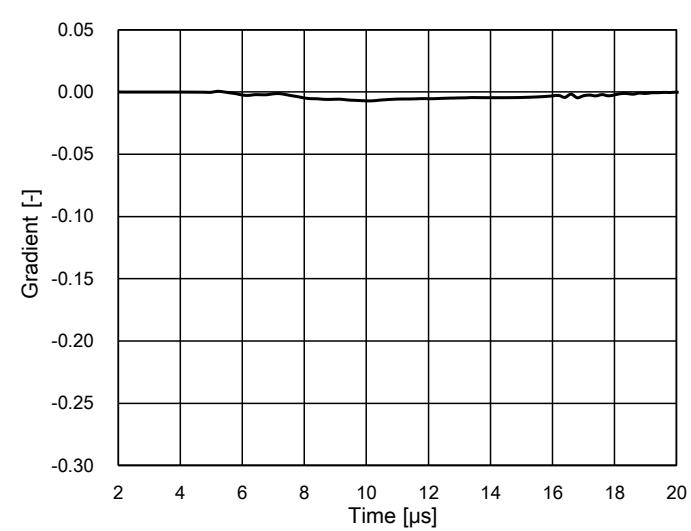

Fig.5-3 Time dependence of the gradient $A_{1}$ of Eq. (4) for $t_{r e f}=2 \mu \mathrm{s}$

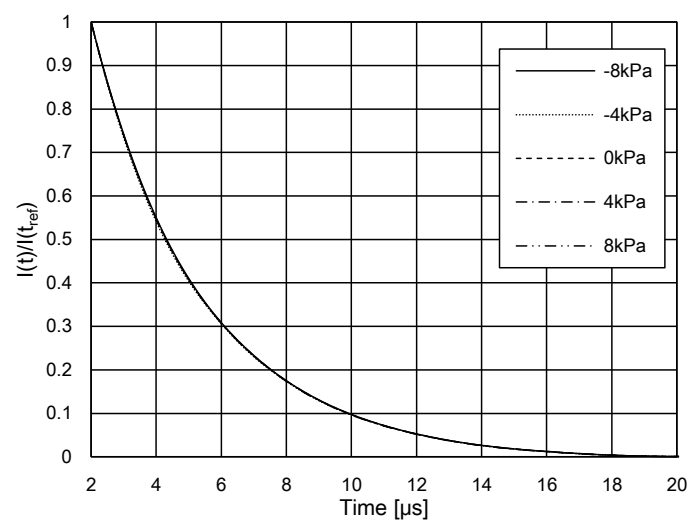

Fig.5-2 Decay characteristics of normalized luminescence intensity for $t_{r e f}=2 \mu \mathrm{s}$

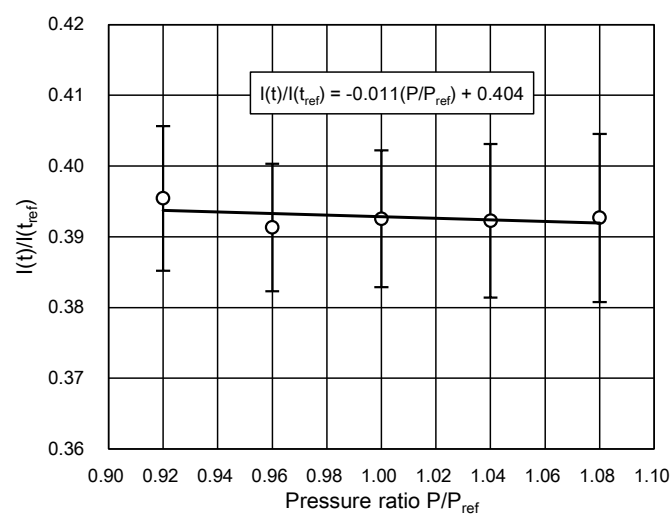

Fig.5-4 Relationship between normalized luminescence $I(t=5 \mu \mathrm{s}) / I\left(t_{\text {ref }}=2 \mu \mathrm{s}\right)$ and normalized pressure

Fig.5 Pressure dependence of mono-layer TSP 


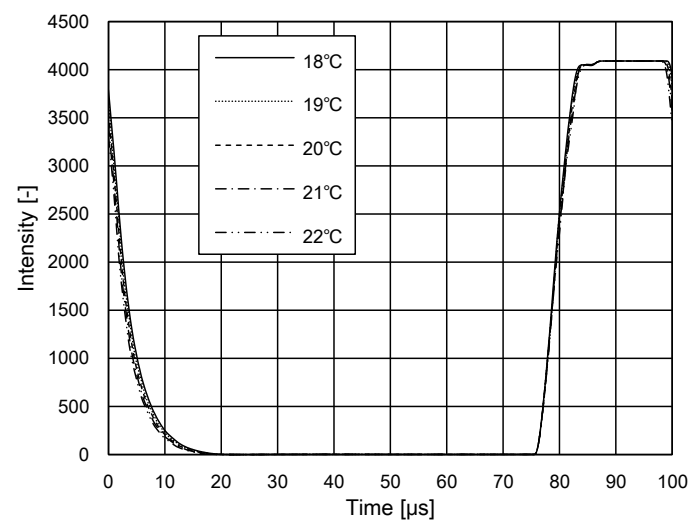

Fig.6-1 Luminescence intensity for a period of irradiation

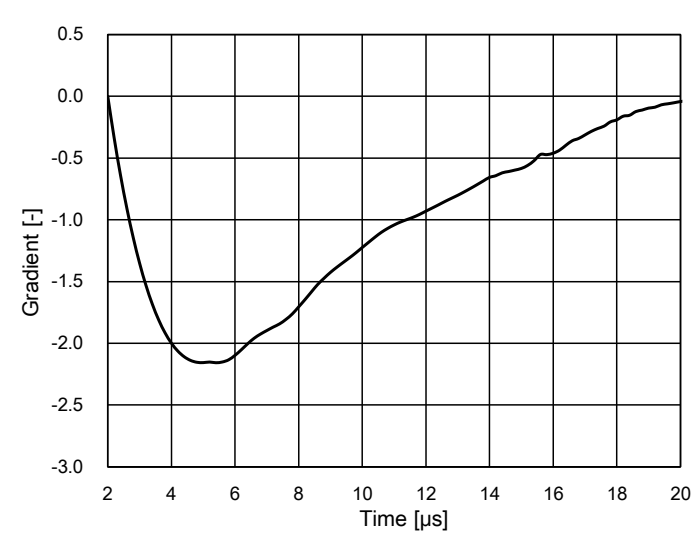

Fig.6-3 Time dependence of the gradient $B_{1}$ of Eq. (5) for $t_{r e f}=2 \mu \mathrm{s}$

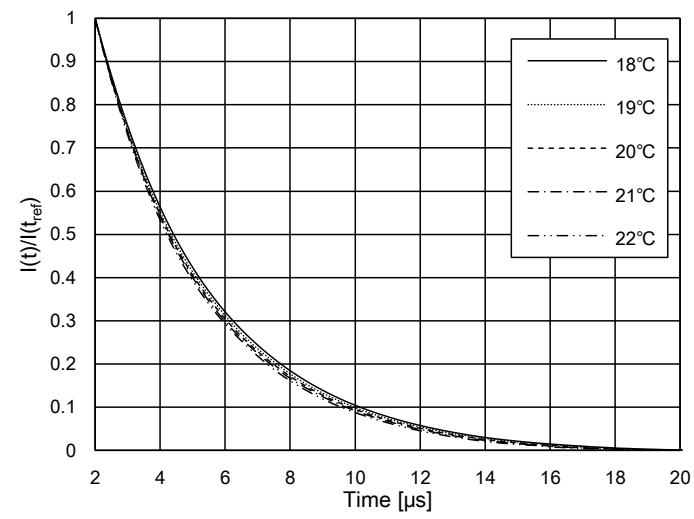

Fig.6-2 Decay characteristics of normalized luminescence intensity for $t_{r e f}=2 \mu \mathrm{s}$

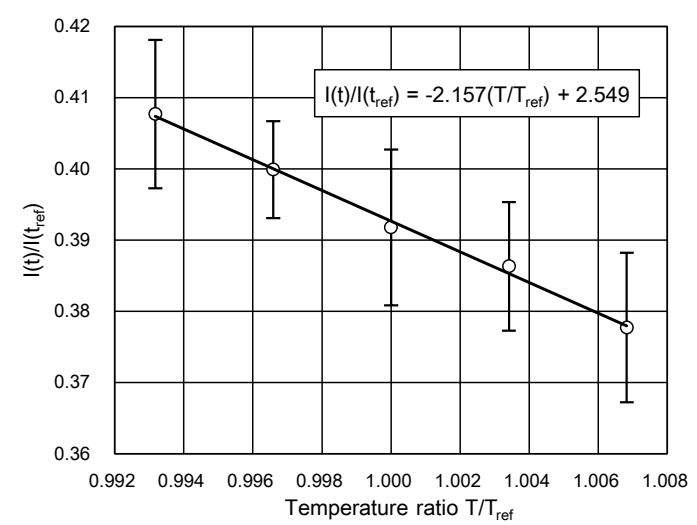

Fig.6-4 Relationship between normalized luminescence $I(t=5 \mu \mathrm{s}) / I\left(t_{r e f}=2 \mu \mathrm{s}\right)$ and normalized temperature

Fig.6 Temperature dependence of mono-layer TSP 


\subsection{Mono-layer PSP}

Fig.7-1 and Fig.8-1 show the dependence of the luminescence intensity of the mono-layer PSP for a period of irradiation, including luminescence decay and excitation, on pressure and temperature, respectively. The luminescence lifetime of the mono-layer PSP is much longer than that of the monolayer TSP. Because the irradiation time per period $(15 \mu \mathrm{s})$ is shorter than the lifetime of PtTFPP, the luminophore of PSP, it is not sufficiently excited, and luminescence intensity of the PSP is lower than that of the TSP. Furthermore, it is shown that the decayed luminescence intensity does not reach zero before the next period of the excitation starts. However, we have confirmed from our preliminary experiments that the property of the decay of normalized luminescence is the same as that for longer period of irradiation and higher duty ratio, for which the luminophore is excited sufficiently and the decayed luminescence intensity reaches zero before the next period of the excitation starts. The intensity of the mono-layer PSP at $t=5 \mu$ s was about half of the intensity of the mono-layer TSP shown in Fig.5-1 and Fig.6-1. Fig.7-2 and Fig.8-2 show the dependence of the luminescence decay characteristics on pressure and temperature for $t_{r e f}=2 \mu \mathrm{s}$. Fig.7-4 and Fig.8-4 show the relationships between normalized luminescence intensity $I(t=26 \mu \mathrm{s}) / I\left(t_{r e f}\right)$ and normalized pressure and temperature, respectively, following to Eq. (4) and (5). It can be confirmed that both pressure and temperature dependence show good linearity and the standard deviation is also smaller than the monolayer TSP. Fig.7-3 and Fig.8-3 show the time dependence of the gradients $A_{1}$ and $B_{1}$ of Eq. (4) and (5), respectively. The gradients $\left|A_{1}\right|$ and $\left|B_{1}\right|$ show the maximum of 0.206 and 2.364, respectively, at $t=26 \mu \mathrm{s}$, that is, $t-t_{r e f}=24 \mu \mathrm{s}$. From the above results, the decay of the luminescence of the PSP has temperature sensitivity as well as pressure sensitivity. The results highlight the significance of temperature compensation for accurate pressure measurement, especially in the condition where the temperature distribution is non-uniform. Considering the application of the PSP to the DL-PTSP as a PSP layer, it is important to consider the method of normalization of the luminescence of the DL-PTSP, especially setting $t_{r e f}$ as the time when the luminescence of the TSP layer of the DL-PTSP completely disappears, to eliminate the effect of the TSP luminescence. Fig.7-5 and Fig.8-5 show the dependence of the luminescence decay characteristics on pressure and temperature, but the reference time is set at $t_{r e f}=26 \mu \mathrm{s}$. The results are almost the same for equal $t-t_{r e f}$, compared to the results for $t_{r e f}=2 \mu \mathrm{s}$ shown in Fig.7-2 and Fig.8-2. Fig.7-6 and Fig.8-6 depict the time dependence of the gradient $A_{1}$ and $B_{1}$ of Eq. (4) and (5), respectively. From Fig.7-6 and Fig.8-6, it is clarified that the gradients $\left|A_{1}\right|$ and $\left|B_{1}\right|$ show the maximum at time $t=51 \mu \mathrm{s}$. In this case, the difference between the time showing the maximum gradient and the reference time is $t-t_{\text {ref }}=25 \mu \mathrm{s}$; this is similar to the case for $t_{r e f}=2 \mu \mathrm{s}$ shown in Fig.7-3 and Fig.8-3. Fig.7-7 and Fig.8-7 show the relationships between normalized luminescence intensity $I(t=51 \mu \mathrm{s}) / I\left(t_{r e f}\right)$ for $t_{r e f}=26 \mu \mathrm{s}$ and normalized pressure and temperature, respectively. It can be confirmed that the sensitivity decreases and the 
standard deviation increases slightly compared with the results shown in Fig.7-4 and Fig.8-4, but it is still high enough. This result suggests that degradation of sensitivity and error has increased because the luminescence intensity of PSP is decreased as reference time $\left(T_{r e f}\right)$ is delayed. However, it is expected that the pressure sensitivity of the DL-PTSP is almost equal to that of the monolayer PSP, by setting as $t_{r e f}=26 \mu$ s to eliminate the contribution of the TSP layer with short lifetime. .

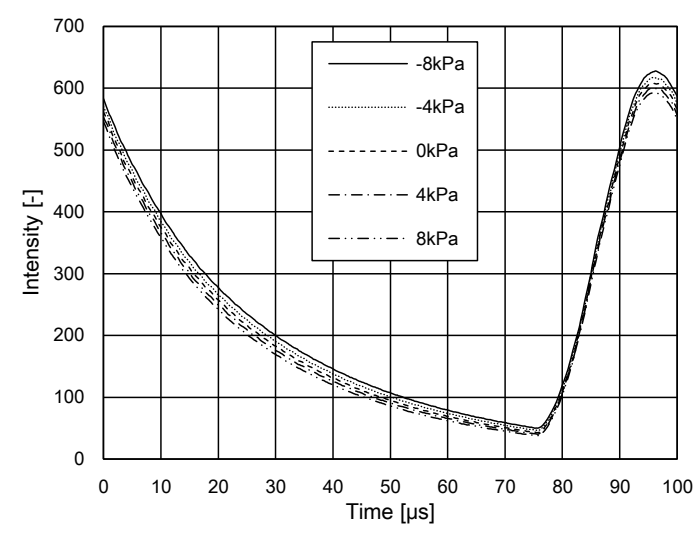

Fig.7-1 Luminescence intensity for a period of irradiation

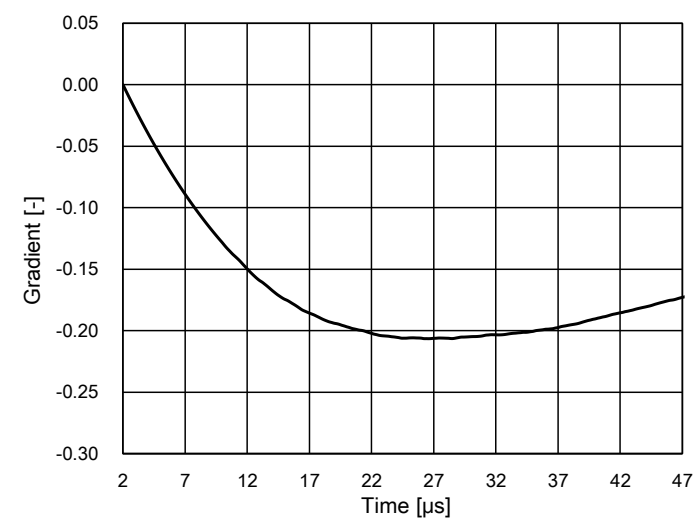

Fig.7-3 Time dependence of the gradient $A_{1}$ of Eq. (4) for $t_{\text {ref }}=2 \mu \mathrm{s}$

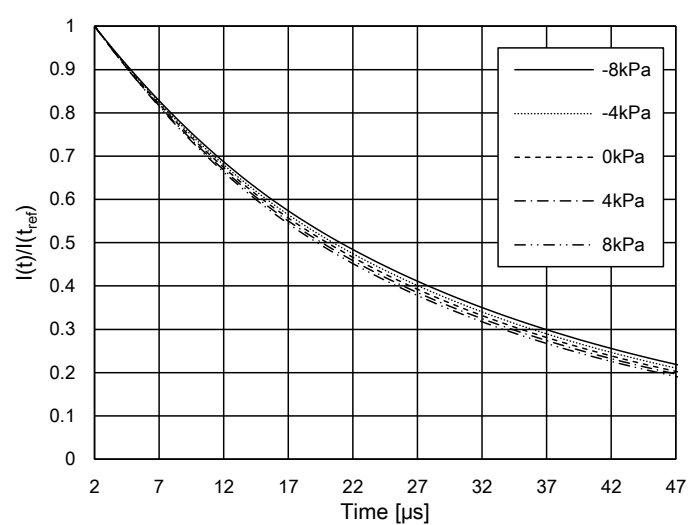

Fig.7-2 Decay characteristics of normalized luminescence intensity for $t_{r e f}=2 \mu \mathrm{s}$

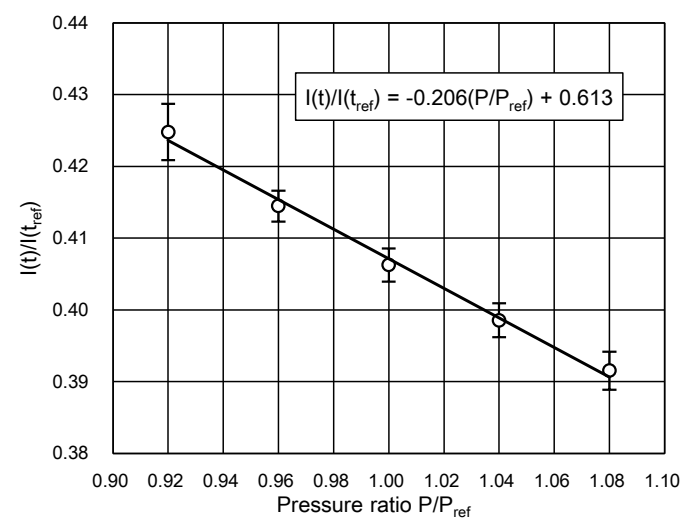

Fig.7-4 Relationship between normalized luminescence $\quad I(t=26 \mu \mathrm{s}) / I\left(t_{r e f}=2 \mu \mathrm{s}\right)$ and normalized pressure 


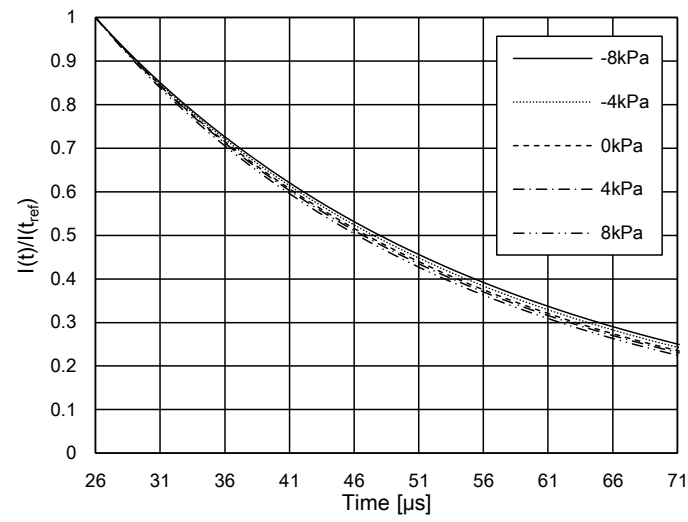

Fig.7-5 Decay characteristics of normalized luminescence intensity for $t_{r e f}=26 \mu \mathrm{s}$

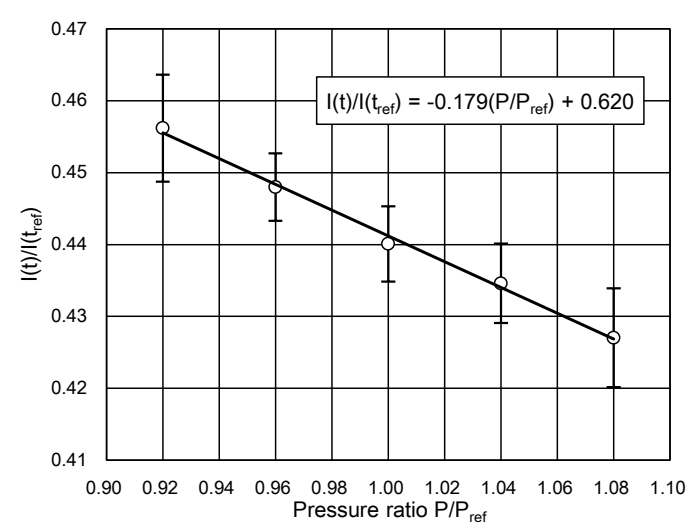

Fig.7-7 Relationship between normalized luminescence $\quad I(t=51 \mu \mathrm{s}) / I\left(t_{\text {ref }}=26 \mu \mathrm{s}\right)$ and normalized pressure

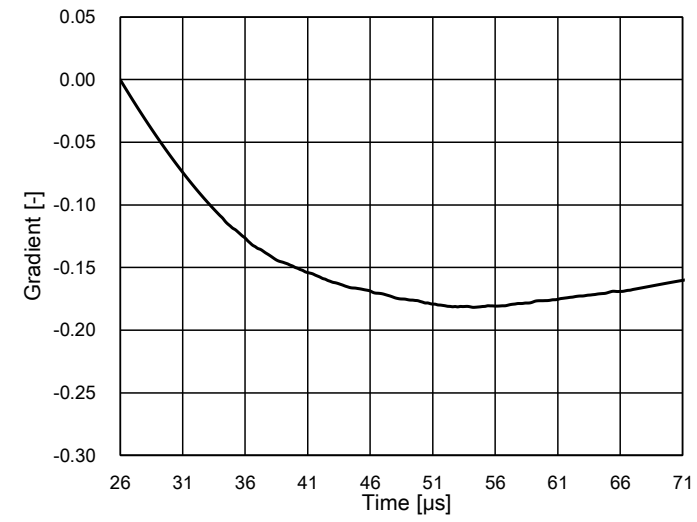

Fig.7-6 Time dependence of the gradient $A_{1}$ of Eq. (4) for $t_{r e f}=26 \mu \mathrm{s}$

Fig.7 Pressure dependence of mono-layer PSP 


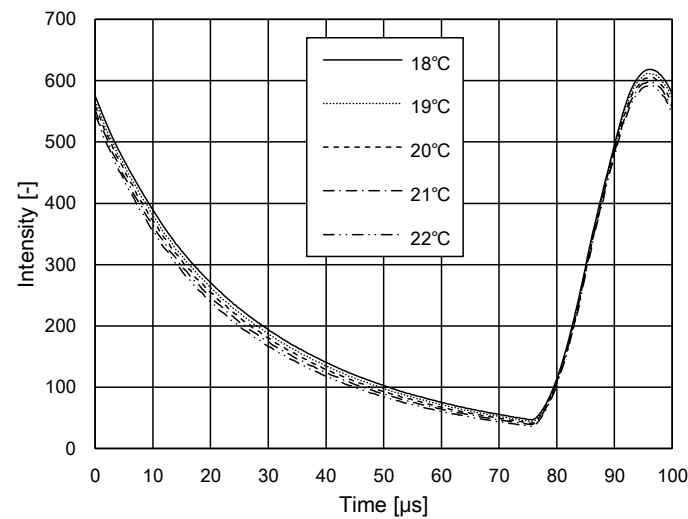

Fig.8-1 Luminescence intensity for a period of irradiation

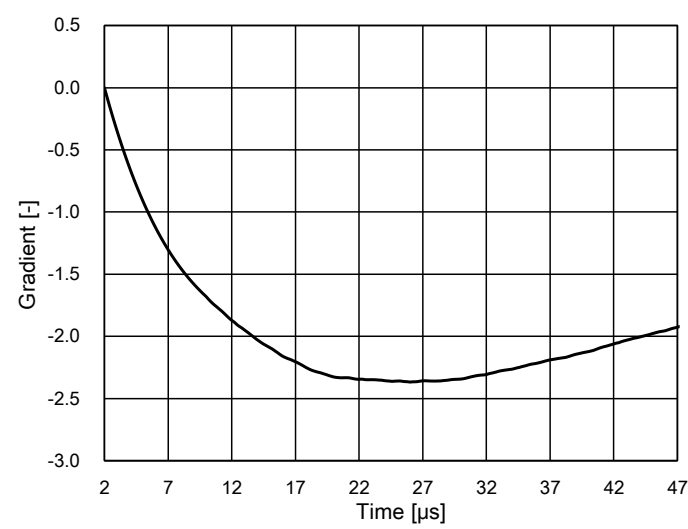

Fig.8-3 Time dependence of the gradient $B_{1}$ of Eq. (5) for $t_{r e f}=2 \mu \mathrm{s}$

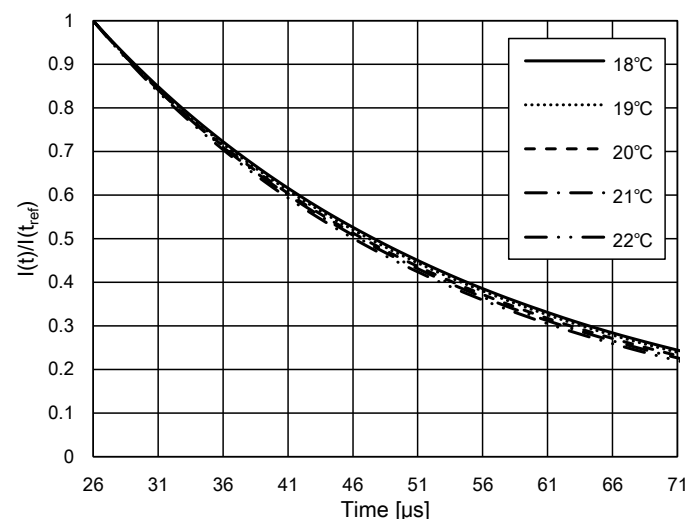

Fig.8-5 Decay characteristics of normalized luminescence intensity for $t_{r e f}=26 \mu \mathrm{s}$

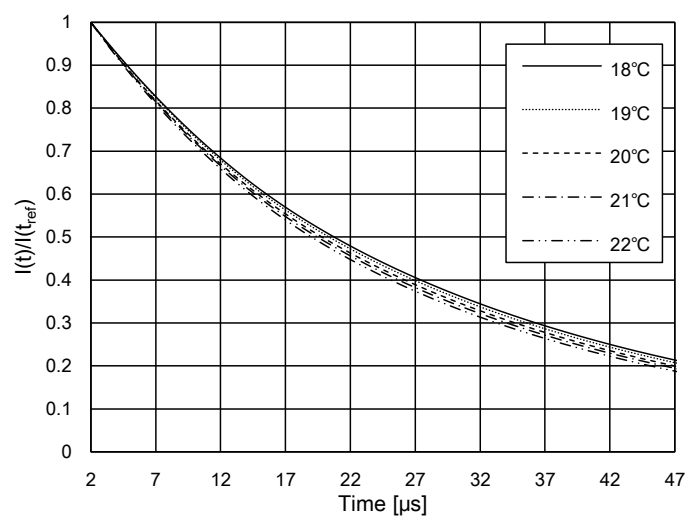

Fig.8-2 Decay characteristics of normalized luminescence intensity for $t_{r e f}=2 \mu \mathrm{s}$

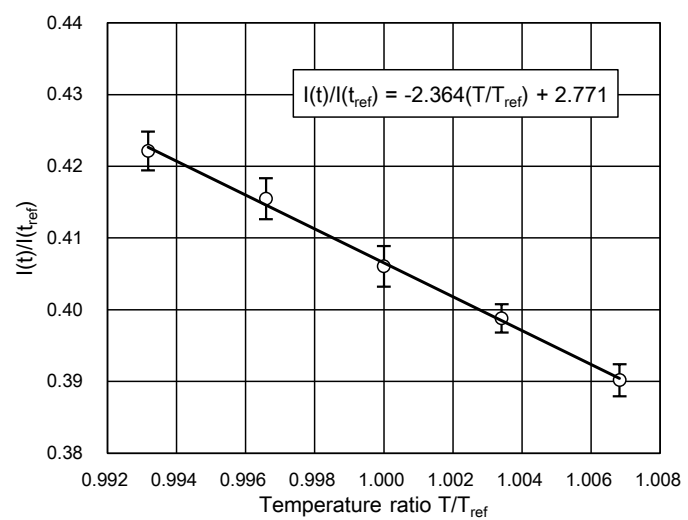

Fig.8-4 Relationship between normalized luminescence $\quad I(t=26 \mu \mathrm{s}) / I\left(t_{\text {ref }}=2 \mu \mathrm{s}\right)$ and normalized temperature

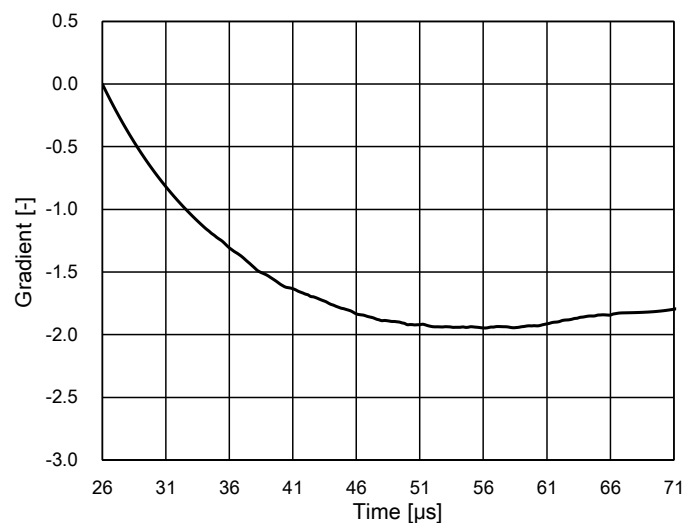

Fig.8-6 Time dependence of the gradient $B_{1}$ of Eq. (5) for $t_{r e f}=26 \mu \mathrm{s}$ 


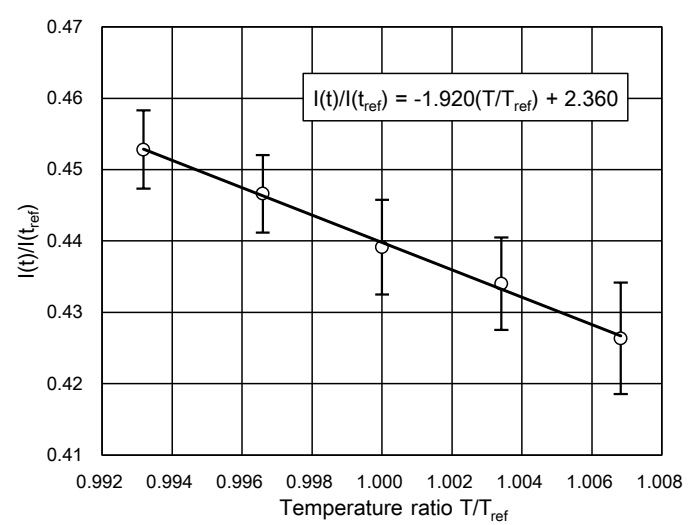

Fig.8-7 Relationship between normalized luminescence $\quad I(t=51 \mu \mathrm{s}) / I\left(t_{r e f}=26 \mu \mathrm{s}\right)$ and normalized temperature

Fig.8 Temperature dependence of mono-layer PSP 


\section{Characteristics of DL-PTSP}

Because the DL-PTSP is composed of the two luminophore layers with different lifetime, the luminescence decay characteristics of the DL-PTSP is multi-exponential, and analysis of the dependence of the luminescence decay of the DL-PTSP on pressure and temperature is complicated. However, the luminophore layers of the DL-PTSP are isolated each other by intermediate layers, and the interference between the luminophore layers is very small. As a result, characterization of the TSP component and the PSP component of DL-PTSP can be achieved by evaluating the results of monolayer samples.

Fig.9 shows decay characteristics of normalized luminescence intensity. Fig.9-1 and Fig.9-2 represent the dependence of pressure and temperature, respectively. At $t=5 \mu \mathrm{s}$, the pressure dependence is very small and temperature dependence can be confirmed. After $t=5 \mu \mathrm{s}$, it can be confirmed that the pressure dependence due to the influence of PSP. Fig.10-1 and Fig.10-2 represent the dependence of normalized luminescence intensity $I(t=5 \mu \mathrm{s}) / I\left(t_{\text {ref }}\right)$ on pressure and temperature ratio, respectively, for $t_{r e f}=2 \mu \mathrm{s}$. From Fig.10-1, the gradient $\left|A_{1}\right|$ of Eq. (4), corresponding to the pressure sensitivity, is 0.027 , which is slightly larger than that of the mono-layer TSP, but still sufficiently small. On the other hand, as shown in Fig.10-2, the gradient $\left|B_{1}\right|$ of Eq. (5) showing the temperature dependence is 0.581 ; this is about one-fourth of that of the mono-layer TSP, but much higher than $\left|A_{1}\right|$, and it shows good linearity. The reasons for the decrease in the sensitivity is due to the simultaneous emission of the PSP layer and the TSP layer of DL-PTSP.

The results indicates that the contribution of the TSP layer to the luminescence decay is dominant for $t \leq 5 \mu \mathrm{s}$, and therefore the dependence of normalized intensity $I(t=5 \mu \mathrm{s}) / I\left(t_{r e f}=2 \mu \mathrm{s}\right)$ on pressure is small enough.

Fig.11 shows the dependence of normalized luminescence intensity $I(t=51 \mu \mathrm{s}) / I\left(t_{\text {ref }}=26 \mu \mathrm{s}\right)$ on pressure ratio for the temperature conditions from $18.0^{\circ} \mathrm{C}$ to $22.0^{\circ} \mathrm{C}(291.2 \mathrm{~K}$ to $295.2 \mathrm{~K})$. It can be confirmed that for each temperature condition the normalized luminescence intensity has linear pressure sensitivity, and the plots for different temperature conditions are almost parallel to each other. The slope is almost similar to that of mono-layer PSP. From the results it is clarified that the luminescence decay properties of the DL-PTSP is same as that of the mono-layer PSP, when the reference time is taken as $26 \mu \mathrm{s}$. Fig. 12 shows a least-square-fitting plane indicating the dependence of $I(t=51 \mu \mathrm{s}) / I\left(t_{r e f}=26 \mu \mathrm{s}\right)$ on pressure and temperature, following to Eq.(6), where $P$ is absolute pressure and $T$ is absolute temperature. In addition $P_{\text {ref }}$ and $T_{\text {ref }}$ are known "reference" absolute pressure and absolute temperature, respectively.

$$
\frac{I(t=51 \mu \mathrm{s})}{I\left(t_{r e f}=26 \mu \mathrm{s}\right)}=-0.158 \frac{P}{P_{\text {ref }}}-2.38 \frac{T}{T_{\text {ref }}}+2.96
$$


By calculating the temperature $T$ from Eq. (5) using $I(t=5 \mu \mathrm{s}) / I\left(t_{r e f}=2 \mu \mathrm{s}\right)$ and putting it to Eq. (6), the pressure $P$ can be obtained using $I(t=51 \mu \mathrm{s}) / I\left(t_{r e f}=26 \mu \mathrm{s}\right)$.

Fig.13 shows spatial uniformity of DL-PTSP. Fig.13-1 shows the normalized intensity for each pixel at $(t=5 \mu \mathrm{s}) / I\left(t_{r e f}=2 \mu \mathrm{s}\right)$. The normalized intensity average value is 0.501 and the standard deviation is 0.002 . Fig13-2 show the normalized intensity for each pixel at $I(t=51 \mu \mathrm{s}) / I\left(t_{\text {ref }}=\right.$ $26 \mu \mathrm{s})$. The normalized intensity average value is 0.425 and the standard deviation is 0.016 .

These results confirm the feasibility of simultaneous measurement of temperature and pressure in low-gauge pressure conditions by the DL-PTSP with lifetime method proposed in this study, using a single camera. However, this method has a disadvantage that it has lower pressure sensitivity than the intensity based method. It will be further developed through the modification of the luminophore.

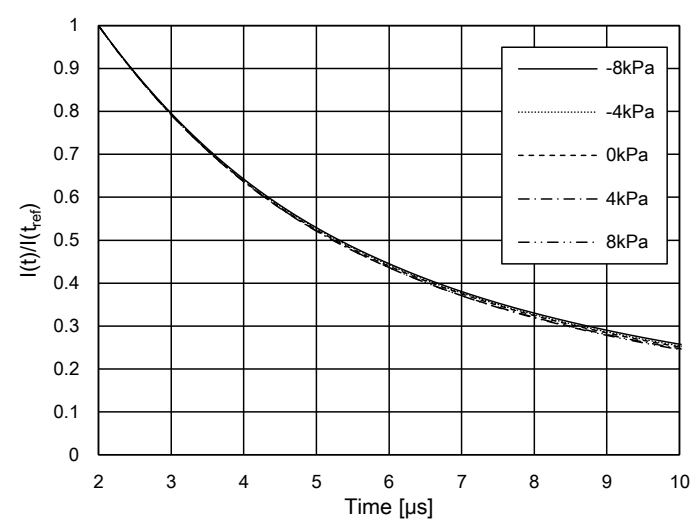

Fig.9-1 Pressure dependence

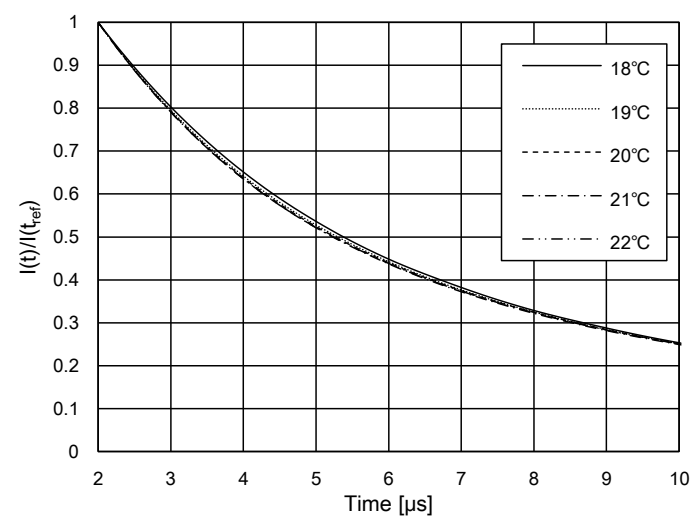

Fig.9-2 Temperature dependence

Fig.9 Decay characteristics of normalized luminescence intensity for $t_{r e f}=2 \mu \mathrm{s}$

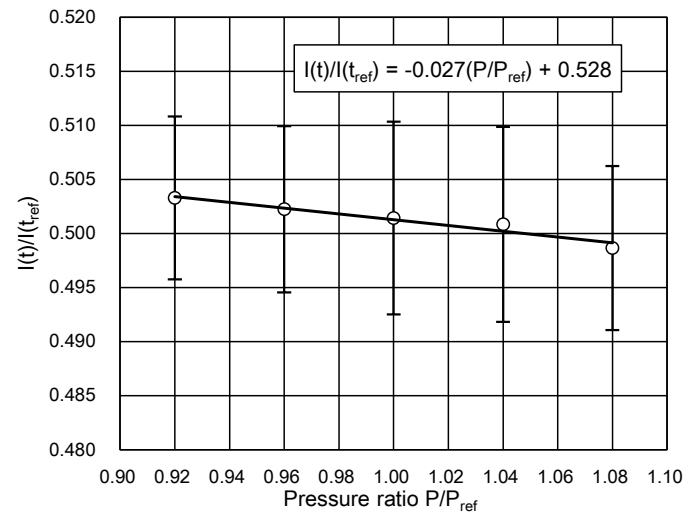

Fig.10-1 Dependence on pressure ratio

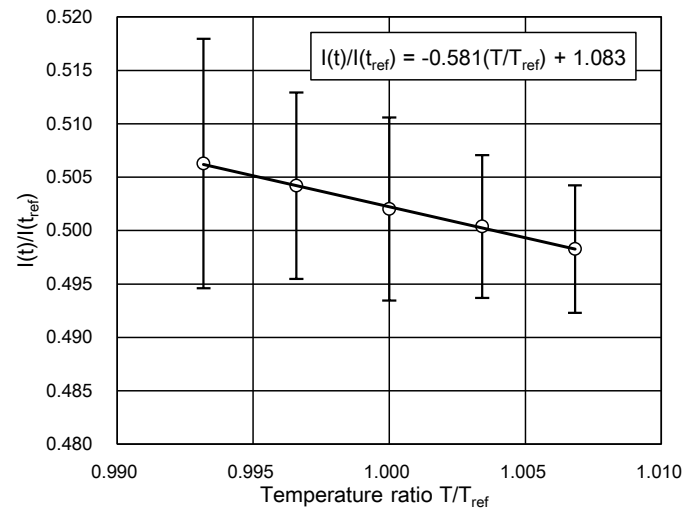

Fig.10-2 Dependence on temperature ratio

Fig.10 Dependence of normalized luminescence intensity $I(t=5 \mu \mathrm{s}) / I\left(t_{r e f}=2 \mu \mathrm{s}\right)$ 


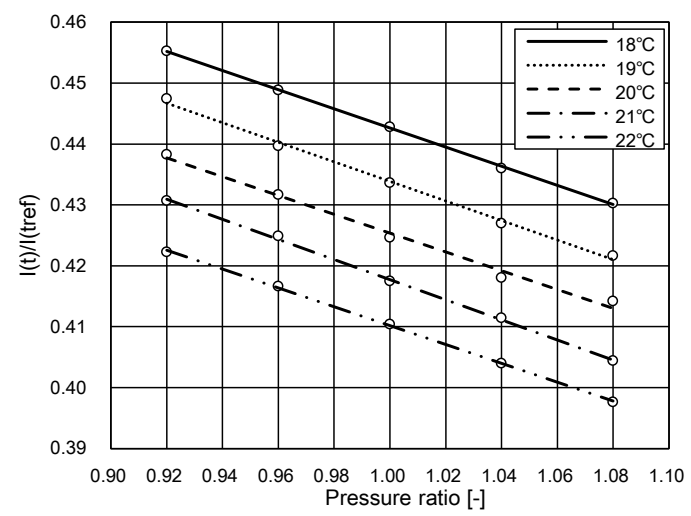

Fig.11 Dependence of normalized luminescence intensity $I(t=51 \mu \mathrm{s}) /$ $I\left(t_{r e f}=26 \mu \mathrm{s}\right)$ on pressure ratio for the

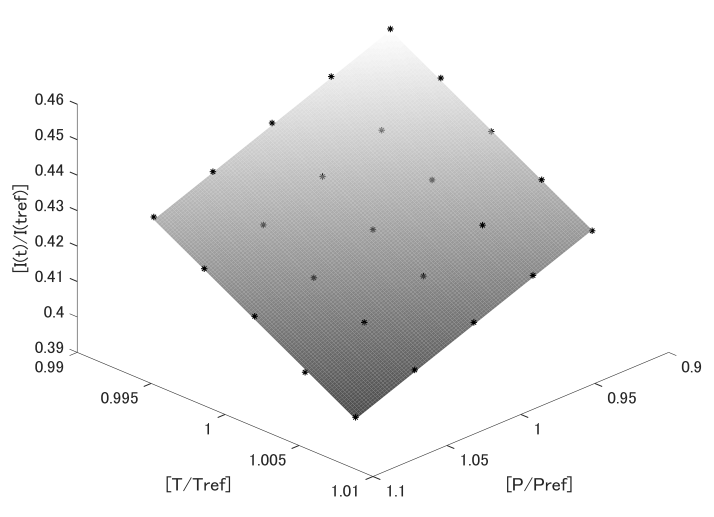

Fig.12 Least-square-fitting plane indicating the dependence of $I(t=51 \mu \mathrm{s}) / I\left(t_{\text {ref }}=\right.$ $26 \mu \mathrm{s})$ on pressure and temperature
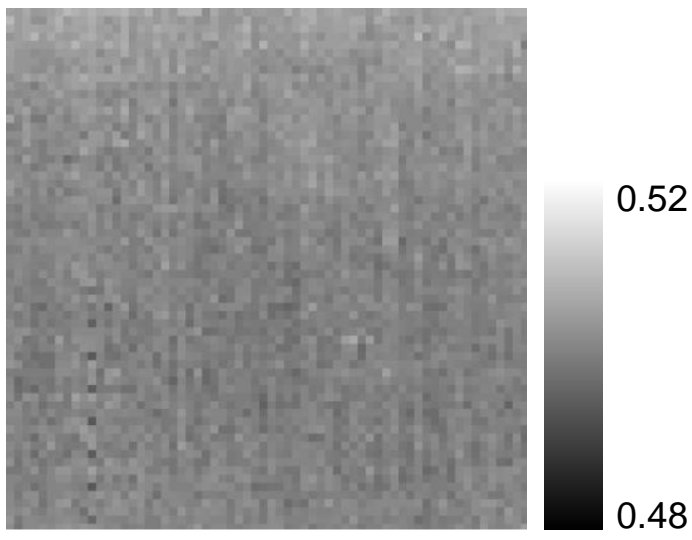

Fig.13-1 $I(t=5 \mu \mathrm{s}) / I\left(t_{\text {ref }}=2 \mu \mathrm{s}\right)$

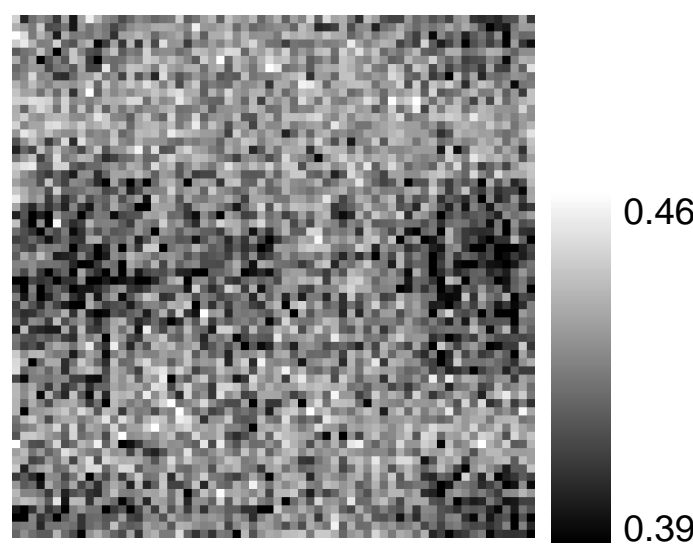

Fig.13-2 $I(t=51 \mu \mathrm{s}) / I\left(t_{r e f}=26 \mu \mathrm{s}\right)$

Fig.13 Spatial uniformity of DL-PTSP 


\section{Conclusion}

In this study, we propose the simultaneous measurement technique of pressure and temperature by DL-PTSP with lifetime-based method, to compensate for the temperature sensitivity of the PSP. The following concluding remarks are obtained.

1. The luminescence intensity of the two luminophore layers of DL-PTSP was optimized for lifetime-based method by setting appropriate irradiation time for one period, to reduce the intensity of the PtTFPP-based PSP layer with longer lifetime while keeping the intensity of the Ru-phen-based TSP layer with shorter lifetime.

2. We examined the excitation and luminescence decay characteristics of luminophore by using a high speed camera, by controlling the irradiation delay of light source.

3. Luminescence intensity of Ru-phen-based mono-layer TSP fall close to zero at $t \sim 20 \mu \mathrm{s}$ and the highest temperature sensitivity can be achieved by taking $I(t) / I\left(t_{r e f}=2 \mu \mathrm{s}\right)$ at $t-t_{r e f}=3 \mu \mathrm{s}$. It is also confirmed that the pressure dependence is negligibly small.

4. Luminescence of PtTFPP-based mono-layer PSP has both pressure and temperature sensitivity, and the sensitivity is the highest by taking $I(t) / I\left(t_{r e f}\right)$ at $t=26 \mu$ s when $t_{r e f}$ is taken at $2 \mu \mathrm{s}$. Because the decay process of the mono-layer PSP is almost exponential, the sensitivity of $I(t) / I\left(t_{r e f}\right)$ with $t_{r e f}=26 \mu \mathrm{s}$ is almost equal to that with $t_{r e f}=2 \mu \mathrm{s}$ if $t-t_{r e f}$ is equal, and the luminescence of Ru-phen-based TSP is disappeared at $t=26 \mu$ s. Therefore, by taking $t_{r e f}=26 \mu \mathrm{s}$, luminescence of the PSP layer of the DL-PTSP can be examined.

5. By the experimental analysis of the DL-PTSP that we have developed, it is clarified that the contribution of the TSP layer to the luminescence decay is dominant for $t \leq 5 \mu \mathrm{s}$, and therefore the dependence of normalized intensity $I(t=5 \mu \mathrm{s}) / I\left(t_{r e f}=2 \mu \mathrm{s}\right)$ of the DL-PTSP on pressure is small enough. On the other hand, the dependence of normalized intensity $I(t=51 \mu \mathrm{s}) /$ $I\left(t_{r e f}=26 \mu \mathrm{s}\right)$ of the DL-PTSP on pressure and temperature is almost the same as that of monolayer PSP. The characteristics of the DL-PTSP confirms that the lifetime-based method for the DL-PTSP can separate the contribution of the luminescence of the TSP component and that of the PSP component. The result enables the simultaneous measurement of temperature and pressure by using DL-PTSP with a single camera. 


\section{Acknowledgments}

This work was supported by Suzuki Foundation and Harada Memorial Foundation. The observation of cross-section of samples was made using SEM(SHIMADZU, SS550) at the central of advanced instrumental analysis, Kyushu University.

\section{Reference}

[1] T. Liu and J. Sullival, Pressure and temperature sensitive paints, Springer, 2005.

[2] T. Liu, B. Campbell, S. Burns and J. Sullivan, "Temperature and Pressure-Sensitive Luminescent Paints in Aerodynamics", American Society of Mechanical Engineers, Appl. Mech. Rev 50(4), pp. 227-246, 1997.

[3] J. Bell, E. Schairer, L. Hand and R. Mehta, "Surface pressure measurements using luminescent coatings", Annual Review of Fluid Mechanics, Vol. 33, pp. 155-206, 2001.

[4] J. Crafton, S. Fonov, R. Forlines and S. Palluconi, "Development of Pressure-Sensitive Paint systems for low speed flows and large wind tunnels", 51st AIAA Aerospace Sciences Meeting including the New Horizons Forum and Aerospace Exposition 2013, ISBN: 978-162410181-6, 2013.

[5] M. Ibrahim, S. Matsumoto, K. Mori and Y. Nakamura, "Visualization of Pressure Field over Rotating Blades Using Pressure Sensitive Foil Technique", Transactions of the Japan Society for Aeronautical and Space Sciences, Volume 53, Issue 182 pp. 243-249, 2011.

[6] D. Peng, S. Wang and Y. Liu, "Fast PSP measurements of wall-pressure fluctuation in lowspeed flows: improvements using proper orthogonal decomposition", Experiments in Fluids, DOI 10.1007/s00348-016-2130-z, 2016.

[7] J. Bell, "Applications of pressure-sensitive paint to testing at very low flow speeds", 42nd AIAA Aerospace Sciences Meeting and Exhibit, AIAA 2004-878, 2004.

[8] H. Jeong, D. Seo, Y. Byun, J. Lee and S. Kim, "Application of PSP to 65-deg Delta Wing at Low Speeds", Proceedings of 2010 Asia-Pacific International Symposium on Aerospace Technology(APISAT 2010), pp. 211-214, 2010.

[9] S. Sano, T. Yuuki, T. Hyakutake, K. Morita, H. Sakaue, S. Arai, H. Matsumoto and T. Michinobu, "Temperature compensation of pressure-sensitive luminescent polymer sensors", Sensors and Actuators B: Chemical, Volume 255, Part 2, pp. 1960-1966, 2018.

[10] K. Mitsuo, M. Kurita, K. Nakakita and S. Watanabe, "Temperature Correction of PSP Measurement for Low-Speed Flow Using Infrared Camera", 21st International Congress on Instrumentation in Aerospace Simulation Facilities, 05 10-1, 30 August, Sendai, Japan, 2005.

[11] K. Nakakita, M. Kurita, K. Mitsuo and S. Watanabe, "Practical pressure-sensitive paint measurement system for industrial wind tunnels at JAXA", Measurement Science and Technology, 17, pp. 359-366, 2006. 
[12] T. Kameya, Y. Matsuda, Y. Egami, H. Yamaguchi and T. Niimi, "Combined Pressure and Temperature Sensor Using Pressure- and Temperature-Sensitive Paints", 2012 international symposium on micro-nanomechatronics and human science(MHS 2012), Article number 6492496, pp. 473-475, 2012.

[13] L. Goss, G. Jones, J. Crafton, and S. Fonov, "Temperature Compensation for Temporal (Lifetime) Pressure Sensitive Paint Measurements", 43rd AIAA Aerospace Sciences Meeting and Exhibit, AIAA 2005-1027, 2005.

[14] D. Mochizuki, S. Tamura, H. Yasutake, T. Kataoka, K. Mitsuo and Y. Wada, "A Photostable BiLuminophore Pressure-Sensitive Paint Measurement System Developed with Mesoporous Silica Nanoparticles", Journal of Nanoscience and Nanotechnology, Volume 13, Issue 4, pp. 2777-2781, 2013.

[15] Y. Egami, J. Ueyama, S. Furukawa, T. Kameya, Y. Matsuda, H. Yamaguchi and T. Niimi, "Development of fast response bi-luminophore pressure-sensitive paint by means of an inkjet printing technique", Measurement Science and Technology, Volume 26, Issue 6, Article number 064004, 2015.

[16] D. Peng and Y. Liu, "A grid-pattern PSP/TSP system for simultaneous pressure and temperature measurements", Sensors and Actuators B: Chemical, Volume 222, Article number 18925, pp. 141-150, 2016.

[17] T. Hyakutake, H. Taguchi, J. Kato, H. Nishide and M. Watanabe, "Luminescent Multi-Layered Polymer Coating for the Simultaneous Detection of Oxygen Pressure and Temperature", Macromolecular Chemistry and Physics, Volume 210, Issue 15, pp. 1230-1234, 2009.

[18] K. Moon, H. Mori, Y. Ambe and H. Kawabata, "Development of Dual-layer PSP/TSP System for Pressure and Temperature Measurements in Low-speed Flow Field", ASME-JSME-KSME 2011 Joint Fluids Engineering Conference(AJK 2011), Volume 1, pp. 2767-2772 2011.

[19] H. Mori, N. Omura, K. Maeda, T. Ohbuchi, S. Nakano, H. Ono and Y. Yanase, "Imaging measurement of the pressure distribution using dual-layer PSP/TSP in the supersonic flow field with shockwaves", 16th International Symposium on Flow Visualization, ISFV 16-1064, 2014.

[20] K. Mitsuo, K. Asai, A. Takahashi and H. Mizushima, "Advanced lifetime PSP imaging system for pressure and temperature field measurement", Measurement Science and Technology, Volume 17, Issue 6, pp. 1282-1291, 2006. 